\title{
European Society of Gastrointestinal Endoscopy (ESGE) Guideline Development Policy
}

Authors

Institutions
J.-M. Dumonceau' ${ }^{1}$, C. Hassan ${ }^{2}$, A. Riphaus ${ }^{3}$, T. Ponchon ${ }^{4}$

Institutions are listed at the end of article.

\section{Bibliography}

Dol http://dx.doi.org/

10.1055/s-0031-1291747

Published online: 27.2 .2012

Endoscopy 2012; 44: 626-629

(c) Georg Thieme Verlag KC

Stuttgart · New York

ISSN 0013-726X

\section{Corresponding author}

J. M. Dumonceau, MD PhD

Division of Gastroenterology and Hepatology

Geneva University Hospitals

Rue Micheli-du-Crest, 24

1205 Geneva

Switzerland

Fax: +41-22-3729366

jmdumonceau@hotmail.com
Numerous scientific publications explore the field of gastrointestinal (GI) endoscopy (a Pubmed search currently yields $>73000$ results). Therefore, guidelines have become an indispensable tool for incorporating up-to-date knowledge into daily clinical care. Since the 1990 s, the issuing of

\section{The ESGE Guideline Committee}

$\nabla$

The ESGE Guideline Committee (GLC) comprises a Chair and committee members who are appointed by the GLC after ESGE Governing Board approval. They include members of the ESGE and of the European Society of Gastroenterology and Endoscopy Nurses and Associates (ESGENA). Representatives from the biomedical industry may be invited to act as advisors but are not part of the GLC. The list of current members is published on the ESGE website. Section leaders are elected for four different interest areas, namely relating to the upper GI tract and the small bowel, to the colon, to the biliopancreatic tree, and to patient sedation and monitoring.

The main tasks of the GLC are to propose topics for new ESGE policy documents, to pursue collaboration with other medical societies, to organize the scientific development of ESGE policy documents, and to participate in their detailed preparation. The GLC manages potential conflicts of interest and ensures that all ESGE policy documents are scientifically sound, based on a thorough review of the literature. Section leaders coordinate the work of groups working on different policy documents and decide on the practical details of updates for ESGE policy documents in their area of expertise. guidelines has been a central task of the European Society of Gastrointestinal Endoscopy (ESGE). Here, the ESGE clarifies the types of policy documents that it issues and the methodology used to produce them, taking into account recent methodological developments.

\section{Types of ESGE policy document}

The ESGE now publishes three types of policy document; references are given for examples of the two types of document that have previously been published:

- Clinical Guidelines provide guidance on disease management and on the clinical implications of technological developments. The topics have a considerable impact on clinical practice in the GI endoscopy community and a sufficiently robust evidence base is available $[1,2]$.

- Position statements express the ESGE position on topics that have a narrower impact on GI endoscopy clinical practice, or for which sufficient evidence is not available, or for which the development methodology described below was not strictly followed.

- Technology reviews clarify endoscopic techniques for those who actually perform endoscopic procedures ("how to do it"). They may be produced following the guideline development methods described below if sufficient data are available [3].

\section{New topics for ESGE policy documents \\ $\nabla$}

Potential topics may be proposed spontaneously or following surveys of ESGE member societies, or by GLC members as well as by members of the Endoscopy journal Editorial Board. The formal procedure consists of sending a standardized form, available at the ESGE website, to the GLC Chair. After searching for any existing up-to-date 
guidelines on the proposed topics, the GLC Chair will present the proposed topics to the ESGE Governing Board, which will select and prioritize them. Criteria for the selection of topics will include the burden of disease, the extent of current variation in practice, the potential to improve outcomes, the avoidance of duplication of effort, and the extent of existing evidence on which to base the policy document [4].

\section{Selection of working group members}

Guideline development groups are formed to draft each guideline. They generally involve 10-15 members, including a leader and, if deemed necessary, a person specialized in the methodology of guideline development. The GLC Chair will invite and brief the leader; the leader will propose experts in the endoscopic field as well as patients' representatives, and experts from other specialties and professions if this is deemed useful to guarantee multidisciplinarity. All participants will be chosen and invited according to their expertise and professional qualifications. After discussion with the GLC Chair, the leader will invite the selected experts to join the working group. The working group will comprise several task forces, each with a leader responsible for organization at his/her level. Participating experts will be introduced to key literature about the methodology used and will sign a declaration regarding any competing interests.

The ESGE will not provide honoraria to members of the working group but it will support administrative and meeting costs, including organization and the travel and accommodation of participants. If the ESGE receives financial support from the biomedical industry or from foundations, for developing a specific policy document, then such support will not be obtained from only a single company. Financial support for the development of ESGE policy documents must not have any conditions. Contributing industries or other entities will not be allowed to have representatives present during meetings held for developing policy documents.

\section{Development of ESGE policy documents}

$\nabla$

\section{Review of the evidence}

The working group will develop a list of key questions according to the PICO format (population, intervention, comparison, and outcome), with series of key questions assigned to task forces [5]. A literature search will then be performed within a range of sources, including the Cochrane Library and Medline at a minimum. The literature search may be done by members of the working group or by one designated person. Key features of the systematic literature review include an explicit search strategy and selection of essential elements according to defined inclusion and exclusion criteria. The resulting evidence will be summarized in evidence tables for the most important outcomes. Evidence tables and the complete search strategy (with sufficient detail to allow reproduction of the search) will be published online as appendixes to the policy document published in the journal Endoscopy.

\section{Grading system}

The GLC, in agreement with the ESGE Governing Board, has decided to adopt the Grading of Recommendations Assessment, Development and Evaluation (GRADE) system for grading evidence levels and recommendation strengths [6]. The GRADE system is clinically oriented as the grading of recommendations depends on the balance between benefits and risks or burden of any health intervention. Differently from other grading systems, the GRADE system has been conceived to allow for dissociation between evidence levels and recommendation strengths. This is related to the clinically oriented assumption that policies should be strongly recommended whenever the benefits clearly outweigh the risks or burden (or vice versa), irrespective of the level of supporting evidence.

According to the GRADE system, the evidence level strictly depends on the likelihood that further research would change our confidence regarding the estimate of the trade-off between the benefit and the risks/burden. The assessment of the evidence lev-

\footnotetext{
Evidence level

$\begin{array}{ll}\text { High quality } & \text { One or more well-designed and well-executed randomized controlled trials (RCTs) that yield } \\ & \text { consistent and directly applicable results. } \\ & \text { This level also means that further research is very unlikely to change our confidence in the } \\ & \text { estimate of effect. } \\ \text { Moderate quality } & \text { RCTs with important limitations (i. e., biased assessment of the treatment effect, large loss to } \\ & \text { follow-up, lack of blinding, unexplained heterogeneity), indirect evidence originating from } \\ & \text { similar (but not identical) populations of interest, and RCTs with a very small number of } \\ & \text { participants or observed events. } \\ & \text { In addition, evidence from well-designed controlled trials without randomization, from well- } \\ & \text { designed cohort or case - control analytic studies, and from multiple time series with or without } \\ & \text { intervention is in this category. } \\ & \text { This level also means that further research will probably have an important impact on our } \\ & \text { confidence in the estimate of effect and may change the estimate. }\end{array}$

${ }^{1}$ Quality of evidence based on observational studies may be rated as moderate or even high, depending on circumstances under which evidence is obtained from observational studies. Factors that may contribute to upgrading the quality of evidence include a large magnitude of the observed effect, a dose-response association, or the presence of an observed effect when all plausible confounders would decrease the observed effect.

${ }^{2}$ Insufficient evidence to determine for or against routinely providing a service.
}

Table 1 Levels of evidence according to the Grading of Recommendations Assessment, Development and Evaluation (GRADE) system [6]. 
Table 2 Strength of recommendations according to the GRADE system [6].

\begin{tabular}{ll}
$\begin{array}{l}\text { Strength of } \\
\text { recommendation }\end{array}$ & $\begin{array}{l}\text { Benefits clearly outweigh risks and burden, or vice } \\
\text { versa. Usually stated as "we recommend." }\end{array}$ \\
\hline Strong & $\begin{array}{l}\text { Benefits closely balanced with risks and burden. } \\
\text { Usually stated as "we suggest." }\end{array}$ \\
\hline
\end{tabular}

el takes into account, apart from study design, the directness of the relationship between the study results and the selected outcome, the consistency across different studies, the evidence of a dose-response gradient, and the limitation or bias of the available studies ( $\bullet$ Table 1 ). When the evidence level is very low, it may provide an insufficient basis for any recommendation to be made.

According to the GRADE system, the recommendation strength depends on four main factors: trade-offs between the benefits and the risks/burden, evidence level, patients' values and preferences, and cost considerations. There are two grades of recommendation: strong and weak ( $\bullet$ Table2). Strong recommendations indicate that benefits clearly outweigh the risks and burden, so that the intervention should be received by most individuals with a particular condition and can be adopted as policy in most circumstances. Weak recommendations indicate that benefits are finely balanced against risks and burden or that a substantial uncertainty exists about the magnitude of benefits and risks. Patient preferences may strongly influence the appropriate intervention. Several medical organizations have proposed that interventions strongly recommended on the basis of the GRADE system may be adopted as reliable performance indicators of the improvement of patient care [7].

\section{Meetings}

Once the evidence tables have been constructed and the evidence statements plus recommendations have been drafted, a face-toface meeting is held in order to: (i) deal with any significant unresolved issues or controversies, and (ii) vote on evidence statements and recommendations.

If $80 \%$ or more of the working group is in agreement, the recommendation will be approved. In the case of disagreement, the leader may decide to continue until a final agreement is reached, to remove the recommendation because of lack of agreement, or to keep the recommendation with an explanation of the disagreement.

\section{Structure of manuscript submitted for review \\ $\nabla$}

The title of the document should specify the topic, the type of ESGE policy document (from those listed above), and the names of the endorsing societies.

A structured abstract of no more than 300 words should be provided, with the following headings: Background, Aims and topics, Associated document (in the case of combined publication, for example a Clinical Guideline and a Technology Review), Methods, Summary of selected recommendations.

The main text should contain at a minimum the following sections: an introduction (specifying target audience and endorsing societies at the end); a methods section; the topic of each task force with statements and recommendations followed by a dis- cussion; a competing interests statement; hyperlinks to online appendixes; references; and tables. Inclusion of algorithms is encouraged. The recommendations felt to be the most important for clinical practice should be in bold characters. The maximum word count for the main text, excluding references, should be 6500.

A separate summary titled "Quick reference guide" should list evidence statements and recommendations, and the algorithms if deemed useful.

Appendixes that will be made available online-only should include: (i) the Quick reference guide; (ii) a table showing each task force topic with its key questions and also the personnel comprising the task force; (iii) the numbers of articles considered and finally used to answer key questions; (iv) the list of terms and of databases used for the literature search to answer the key questions, with sufficient detail to allow reproduction of the search; and (v) for each important key question, an evidence table listing each study included in the analysis with its most relevant findings and statistical data.

As a legal disclaimer, the following explanation about the use of ESGE policy documents has been included in recent ESGE Guidelines:

"ESGE Guidelines represent a consensus of best practice based on the available evidence at the time of preparation. They might not apply in all situations and should be interpreted in the light of specific clinical situations and resource availability. Further controlled clinical studies may be needed to clarify aspects of these statements, and revision may be necessary as new data appear. Clinical considerations may justify a course of action at variance with these recommendations. ESGE Guidelines are intended to be an educational device for providing information that may assist endoscopists in providing care to patients. They are not rules and should not be construed as establishing a legal standard of care or as encouraging, advocating, requiring, or discouraging any particular treatment."

Because of space constraints, in future Guidelines this disclaimer will be noted and referenced but not reproduced in full.

\section{Peer review}

$\nabla$

The peer review process for ESGE policy documents will be as follows: when a topic is selected, two peer reviewers will be chosen from the ESGE Governing Board; these peer reviewers will be notified one month before the manuscript is submitted, to ensure that the peer review process takes no more than two weeks. If the required expertise is not available amongst ESGE Governing Board members, peer review will be sought from outside of the ESGE Governing Board.

One peer reviewer will check the manuscript using the Appraisal of Guidelines, Research and Evaluation (AGREE) instrument [8]. Briefly, the AGREE instrument consists of a validated 23-item tool targeting six quality-related domains (scope and purpose, stakeholder involvement, rigor of development, clarity and presentation, applicability, editorial independence). 


\section{Dissemination and adaptation}

\section{$\nabla$}

All ESGE policy documents will be made freely available from the ESGE website, in addition to publication in the journal Endoscopy. As representatives of various countries have indicated the desire to adapt ESGE policy documents at their national level, it has been decided by the ESGE Governing Board and Thieme representatives that this facility would be automatically granted with no fee, after a formal request to the ESGE Governing Board. In its title, the adapted policy document should include the title (in English) of the original ESGE policy document and the names of the authors of the original document. Any modifications of the original document, apart from translation, should be clearly identified in the adapted version of the document.

\section{Update of ESGE policy documents}

The date scheduled for document revision will be stated in each ESGE policy document. If new evidence becomes available that would significantly modify recommendations, updates to the policy document in the interim period will be noted on the ESGE website.

\section{Competing interests: None}

\footnotetext{
Institutions

${ }^{1}$ Division of Gastroenterology and Hepatology, Geneva University Hospitals, Geneva, Switzerland

2 Digestive Endoscopy Unit, Catholic University, Rome, Italy

${ }^{3}$ Department of Medicine, Ruhr University Bochum, Bochum, Germany

${ }^{4}$ General Secretary of the European Society of Gastrointestinal Endoscopy (ESGE) Department of Hepatogastroenterology, Hôpital Edouard Herriot, Lyon, France
}

\section{Acknowledgment \\ $\nabla$}

The authors thank Michael Bretthauer for helpful comments about the structure of manuscripts to be submitted and Hilary Hamilton-Gibbs for revising this manuscript.

\section{References}

1 Dumonceau J-M, Andriulli A, Devière J. European Society of Gastrointestinal Endoscopy (ESGE). et al. Guideline: prophylaxis of post-ERCP pancreatitis. Endoscopy 2010; 42: 503-515

2 Dumonceau J-M, Polkowski M, Larghi A et al. Indications, results, and clinical impact of endoscopic ultrasound (EUS)-guided sampling in gastroenterology: European Society of Gastrointestinal Endoscopy (ESGE) Clinical Guideline. Endoscopy 2011; 43: 897-912

3 Polkowski M, Larghi A, Weynand B et al. Learning, techniques and complications of endoscopic ultrasound-guided sampling in gastroenterology: ESGE Technical Guideline. Endoscopy 2012; 44: 190-206 Epub 2011 Dec16

4 Scottish Intercollegiate Guidelines Network. SIGN 50: A guideline developer's handbook. Available at: http://wwwsignacuk/guidelines/ fulltext/50/indexhtml (Accessed on December 2 2010)

5 Bergus GR, Randall CS, Sinift SD et al. Does the structure of clinical questions affect the outcome of curbside consultations with specialty colleagues? Arch Fam Med 2000; 9: 541-547

6 GRADE Working Group. Grading quality of evidence and strength of recommendations. BMJ 2004; 328: 1490

7 Baumann MH, Lewis SZ, Gutterman D. American College of Chest Physicians. ACCP evidence-based guideline development: a successful and transparent approach addressing conflict of interest, funding, and patient-centered recommendations. Chest 2007; 132: 1015-1024 (Epub 2007 May 31)

8 AGREE Collaboration. Development and validation of an international appraisal instrument for assessing the quality of clinical practice guidelines: the AGREE project. Qual Saf Health Care 2003; 12: 18-23 